\title{
Analytical scheme for the solution of multi-higher Fractional Order Linear Integro-Differential Equations of Fredholm type with Variable Coefficients using Adomian and Modified Adomian Decomposition Methods
}

\author{
Shazad Shawki Ahmed", Dashne Chapuk Zahir ${ }^{2}$
}

1 Department of Mathematics, Sulaimani University, Sulaimany ah, Iraq

2 Department of Mathematics, Koya University, Koya, Iraq

\begin{abstract}
In this paper, Adomian decomposition method (ADM) and modified Adomian decomposition method (MADM) has been introduced to find the exact or approximate solution to a wide class of multi-higher fractional order linear integro-differential problems of Fredholm type with variable coefficients in which the fractional derivative is described in the Caputo sense. In this methods the solution of a functional equation is considered as the sum of infinite series of components usually converging to the solution based on the appearance of the noise terms, where a closed form solution is not obtainable, a truncated number of terms is usually used for numerical purposes. Finally, Numerical experiment prepared that modified Adomian decomposition solutions converges faster than Adomian decomposition solution and by several examples illustrate these considerations.
\end{abstract}

\section{Introduction}

In this paper, we consider the multi-higher order Linear Fredholm Integro-Fractional Differential Equations (LFIFDE's) in Caputo sense with variable coefficients in the general form:

$$
\begin{gathered}
{ }_{a}^{C} D_{t}^{\alpha} n(t)+\sum_{i=1}^{n-1} P_{i}(t){ }_{a}^{C} D_{t}^{\alpha}{ }^{\alpha-i} u(t)+P_{n}(t) u(t)=f(t)+\lambda \int_{a} \sum_{j=0}^{m} \mathcal{K}_{j}(t, s){ }_{a}^{C} D_{s}^{\beta_{m-j}} u(s) d s, \\
\quad a \leq t \leq b \quad \ldots(1)
\end{gathered}
$$

Subject to the conditions:

$$
\mathcal{H}_{\ell}\left(u^{(0)}\left(c_{\xi}\right), u^{(1)}\left(c_{\xi}\right), \cdots, u^{(\ell)}\left(c_{\xi}\right)\right)=C_{\ell} ; c_{\xi} \in[a, b], \forall \xi
$$

for all $\ell=0,1, \cdots, \mu-1 ; \mu=\max \left\{\left\lceil\alpha_{n}\right\rceil,\left\lceil\beta_{m}\right\rceil\right\}$ where $\mathcal{H}_{\ell}$ are the linear combination of $u^{(0)}\left(c_{\xi}\right), u^{(1)}\left(c_{\xi}\right), \cdots, u^{(\mu-1)}\left(c_{\xi}\right)$; where $\alpha_{i}, \beta_{j} \in \mathbb{R}^{+}$for all $(i, j \neq 0), m_{\alpha_{i}}-1<\alpha_{i} \leq m_{\alpha_{i}}$ and $m_{\beta_{j}}-$ $1<\beta_{j} \leq m_{\beta_{j}}$ where $m_{\alpha_{i}}=\left\lceil\alpha_{i}\right\rceil$ and $m_{\beta_{j}}=\left\lceil\beta_{j}\right\rceil$ for all $i=1,2, \ldots, n$ and $j=1,2, \ldots, m$. Combine with property that:

$\alpha_{n}>\alpha_{n-1}>\cdots>\alpha_{1}>\alpha_{0}=0$ and $\beta_{m}>\beta_{m-1}>\cdots>\beta_{1}>\beta_{0}=0$.

Denotes the given continuous functions, $u(t)$ is the unknown function, which is the solution of equation (1) and $\lambda$ is a scalar parameter. Where ${ }_{a}^{C} D_{t}^{\gamma} u(t)$ is Caputo's fractional derivative and $\gamma$ is the order of the fractional derivatives.

Adomian decomposition method was introduced by Adomian [3,7] which is an effective technique that has been employed by many researchers to solve scientific problems $[5,6,8,14]$. This method is series solution technique while the solution is viewed as the infinite series sum with easily computed components which converges rapidly to specific solution [7]. In this article we intend to give a new procedure and modifications: ADMs for the first time for solving the class of the multi-high order fractional linear integro-differential equations of Fredholm type.

From here, we present the necessary information's from fractional calculus (FC), Adomian decomposition method (ADM) and modification techniques of ADM, this information's with some important lemmas are used in our suggested procedure to solve our problem (1). 
2. Fractional Calculus:

In this section, we present some basic definitions, lemmas and properties about linear operators of fractional integration and fractional differentiation which are used throughout this paper. For more details, see $[4,9,11,12,15]$ :

\section{Definition 1:}

A real valued function $u$ defined on $[a, b]$ be in the space $C_{\delta}[a, b], \delta$-any real number, if there exists a real number $k>\delta$, such that $u(t)=(t-a)^{k} u_{1}(t)$, where $u_{1} \in C[a, b]$, and it is said to be in the space $C_{\delta}^{n}[a, b]$ if and only if $u^{(n)} \in C_{\delta}[a, b], n$-positive integer number with zero.

\section{Definition 2:}

Let $u \in C_{\delta}[a, b], \delta \geq-1$ with any positive real number $\alpha$. Then the Riemann-Liouville fractional integral operator ${ }_{a} J_{t}^{\alpha}$ of order $\alpha$ of a function $u$, is defined as:

$$
{ }_{a} J_{t}^{\alpha} u(t)= \begin{cases}\int_{a}^{t} \frac{(t-\xi)^{\alpha-1}}{\Gamma(\alpha)} u(\xi) d \xi, & \alpha>0 \\ u(t) \quad \text { whenever } & \alpha=0\end{cases}
$$

Furthermore, for all $\alpha \geq 0, \beta \geq 0$ and $u(t) \in C_{\delta}[a, b], \delta \geq-1$, then:

$$
{ }_{a} J_{t}^{\alpha}{ }_{a} J_{t}^{\beta} u(t)={ }_{a} J_{t}^{\beta}{ }_{a} J_{t}^{\alpha} u(t)={ }_{a} J_{t}^{\alpha+\beta} u(t)
$$

And, for $\alpha \geq 0$ and $\beta>-1$, Define $u$ by $u(t)=(t-a)^{\beta}$ the R-L integratel can be formed as

\section{Definition 3:}

$$
{ }_{a} J_{t}^{\alpha} u(t)=\frac{\Gamma(\beta+1)}{\Gamma(\beta+\alpha+1)}(t-a)^{\beta+\alpha}
$$

Let $\alpha \geq 0$, and $m=\lceil\alpha\rceil$. the Riemann-Liouville fractional derivative operator ${ }_{a}^{R} D_{t}^{\alpha}$, of order $\alpha$ and $u \in C_{-1}^{m}[a, b]$ and defined as:

$$
{ }_{a}^{R} D_{t}^{\alpha} u(t)=\left\{\begin{array}{l}
D_{t}^{m}\left[{ }_{a} J_{t}^{m-\alpha} u(t)\right], \quad \alpha>0 \\
u(t) \quad \text { whenever } \quad \alpha=0 \\
u^{(m)}(t), \text { If } \alpha=m(\in \mathbb{N}) \text { and } u \in C^{m}[a, b]
\end{array}\right.
$$

The RL-derivative for any power function $u(t)=(t-a)^{\beta}$, where $\alpha \geq 0$ and $\beta>-1$ can be express by:

\section{Definition 4:}

$$
{ }_{a}^{R} D_{t}^{\alpha} u(t)=\frac{\Gamma(\beta+1)}{\Gamma(\beta-\alpha+1)}(t-a)^{\beta-\alpha}
$$

The Caputo fractional derivative operator ${ }_{a}^{C} D_{t}^{\alpha}$ of order $\alpha \in \mathbb{R}^{+}$of a function $u \in C_{-1}^{m}[a, b]$ and $m-1<\alpha \leq m, m \in \mathbb{N}$ is defined as:

$$
{ }_{a}^{C} D_{t}^{\alpha} u(t)= \begin{cases}a J_{t}^{m-\alpha}\left[D_{t}^{m} u(t)\right], & \alpha>0 \\ u(t) \quad \text { whenever } \quad \alpha=0 \\ u^{(m)}(t), \text { If } \alpha=m(\in \mathbb{N}) \text { and } u \in C^{m}[a, b]\end{cases}
$$

For $\alpha \geq 0, \alpha \notin \mathbb{N}$ and $m=\lceil\alpha\rceil$ then ${ }_{a}^{C} D_{t}^{\alpha} u(t)$ is continuous on $[a, b]$, and $\left[{ }_{a}^{C} D_{t}^{\alpha} u(t)\right]_{t=a}=0$. Moreover, The ${ }_{a}^{C} D_{t}^{\alpha},(\alpha \geq 0$ and $m-1<\alpha \leq m)$ of the power function $u(t)=(t-a)^{\beta}$ for some $\beta \geq 0$ can be formulated as:

$$
{ }_{a}^{c} D_{t}^{\alpha} u(t)= \begin{cases}0 & \text { if } \beta \in\{0,1,2, \cdots, m-1\} \\ \frac{\Gamma(\beta+1)}{\Gamma(\beta+1-\alpha)}(t-a)^{\beta-\alpha} & \text { if } \beta \in \mathbb{N} \text { and } \beta \geq m \\ \text { or } \beta \notin \mathbb{N} \text { and } \beta>m-1\end{cases}
$$

Hence, we have the following properties:

$$
\begin{array}{ll}
\text { - } & { }_{a}^{R} D_{t}^{\alpha} \mathcal{C}=\mathcal{C} \frac{(t-a)^{-\alpha}}{\Gamma(1-\alpha)} \text { and }{ }_{a}^{C} D_{t}^{\alpha} \mathcal{C}=0 ; \mathcal{C} \text { is any constant; }(\alpha \geq 0, \alpha \notin \mathbb{N}) \\
\text { - } & { }_{a}^{R} D_{t}^{\alpha} u(t)=D_{t}^{m}{ }_{a} J_{t}^{m-\alpha} u(t) \neq{ }_{a} J_{t}^{m-\alpha} D_{t}^{m} u(t)={ }_{a}^{C} D_{t}^{\alpha} u(t) ; m=\lceil\alpha\rceil .
\end{array}
$$

- Let $\alpha \geq 0, m=\lceil\alpha\rceil$ and $u \in C^{m}[a, b]$, then, the relation between the Caputo derivative and R-L integral are formed:

$$
\begin{gathered}
{ }_{a}^{c} D_{t}^{\alpha}\left[{ }_{a} J_{t}^{\alpha} u(t)\right]=u(t) ; \quad a \leq t \leq b \\
{ }_{a} J_{t}^{\alpha}\left[{ }_{a}^{C} D_{t}^{\alpha} u(t)\right]=u(t)-\sum_{k=0}^{m-1} \frac{u^{(k)}(a)}{k !}(t-a)^{k}
\end{gathered}
$$

We adopt Caputo's definition, which is a modification of the R-L definition and has the advantage of dealing properly with initial value problem, for the concept of the fractional derivative. 
Lemma (1): (The Caputo derivative is left inverse of the RL-integral but not right inverse) [10, 13]

i. If $u$ is continuous and $\alpha \geq 0$ with $m-1<\alpha \leq m(\in \mathbb{N})$, then

$$
{ }_{a}^{C} D_{t}^{\alpha}{ }_{a} J_{t}^{\alpha} u(t)=u(t), \quad a \leq t \leq b
$$

ii. Assume that $\alpha \geq 0, m=\lceil\alpha\rceil$, and $u \in C^{m}[a, b]$. Then

$$
{ }_{a} J_{t}^{\alpha}{ }_{a}^{C} D_{t}^{\alpha} u(t)=u(t)-\sum_{k=0}^{m-1} \frac{u^{(k)}(a)}{k !}(t-a)^{k}
$$

\section{Lemma (2):}

If $G$ be a continuous real valued function on $[a, b] \times[a, b]$, then for each non-negative fractional order $m-1<\alpha \leq m(\in \mathbb{N})$ the following are valid:

$$
{ }_{a} J_{t}^{\alpha} \int_{a}^{b} G(t, s) d s=\int_{a}^{b}\left[{ }_{a} J_{t}^{\alpha} G(t, s)\right] d s
$$

and

$$
{ }_{a}^{c} D_{t}^{\alpha} \int_{a}^{b} G(t, s) d s=\int_{a}^{b}\left[{ }_{a}^{c} D_{t}^{\alpha} G(t, s)\right] d s
$$

\section{Proof:}

(i) Applying R-L fractional integral of order $\alpha \geq 0$, definition (2), to integral part $\int_{a}^{b} G(t, s) d s$ for all $t \in[a, b]$ and using the interchanging order of integration, we have:

$$
\begin{aligned}
a J_{t}^{\alpha} \int_{a}^{b} G(t, s) d s=\frac{1}{\Gamma(\alpha)} \int_{a}^{t}(t-\xi)^{\alpha-1}\left[\int_{a}^{b} G(\xi, s) d s\right] d \xi \\
\quad=\frac{1}{\Gamma(\alpha)} \int_{a}^{b} \int_{a}^{t}(t-\xi)^{\alpha-1} G(\xi, s) d \xi d s=\int_{a}^{b}\left[{ }_{a} J_{t}^{\alpha} G(t, s)\right] d s
\end{aligned}
$$

(ii) Applying $\alpha$-Caputo derivative, definition (4) for $m=\lceil\alpha\rceil$, to integral part $\int_{a}^{b} G(t, s) d s$ for all $t \in[a, b]$ and using generalization of the fundamental theorem of integral calculus [1] with the interchanging order of integration, yields:

$$
\begin{aligned}
{ }_{a}^{C} D_{t}^{\alpha} \int_{a}^{b} G(t, s) d s & =\frac{1}{\Gamma(m-\alpha)} \int_{a}^{t}(t-\xi)^{m-\alpha-1}\left(\frac{d}{d \xi}\right)^{m}\left[\int_{a}^{b} G(\xi, s) d s\right] d \xi \\
& =\frac{1}{\Gamma(m-\alpha)} \int_{a}^{t}(t-\xi)^{m-\alpha-1} \int_{a}^{b} \frac{\partial^{m}}{\partial \xi^{m}} G(\xi, s) d s d \xi \\
& =\int_{a}^{b}\left[\frac{1}{\Gamma(m-\alpha)} \int_{a}^{t}(t-\xi)^{m-\alpha-1} \frac{\partial^{m}}{\partial \xi^{m}} G(\xi, s) d \xi\right] d s=\int_{a}^{b}\left[{ }_{a}^{C} D_{t}^{\alpha} G(t, s)\right] d s
\end{aligned}
$$

\section{Lemma (3):}

Assume that $\alpha$ and $\beta$ are two non-negative fractional order such that $\alpha \geq 1$ and $\alpha \geq \beta$ with $m_{\alpha}=\lceil\alpha\rceil, m_{\beta}=\lceil\beta\rceil$ and $u \in C_{-1}^{m}[a, b]$ and $m=\max \left\{m_{\alpha}, m_{\beta}\right\}$. Then

\section{Proof:}

$$
{ }_{a}^{C} D_{t}^{\beta}{ }_{a} J_{t}^{\alpha} u(t)={ }_{a} J_{t}^{\alpha-\beta} u(t), \quad a \leq t \leq b
$$

Using the definition of $\beta$-Caputo fractional derivative (4) with R-L fractional integral (2) for $\alpha$ order, and after apply the generalization of the fundamental theorem of integral calculus [1], yields

$$
{ }_{a}^{c} D_{t}^{\beta}\left[{ }_{a} J_{t}^{\alpha} u(t)\right]={ }_{a} J_{t}^{m_{\beta}-\beta}\left\{D_{t}^{m_{\beta}}\left[{ }_{a} J_{t}^{\alpha} u(t)\right]\right\}={ }_{a} J_{t}^{m_{\beta}-\beta}\left\{\frac{d^{m}}{d t^{m_{\beta}}}\left[\frac{1}{\Gamma(\alpha)} \int_{a}^{t}(t-\xi)^{\alpha-1} u(\xi) d \xi\right]\right\}
$$




$$
\begin{aligned}
={ }_{a} J_{t}^{m_{\beta}-\beta}\left\{\frac{1}{\Gamma(\alpha)}\right. & \left.(\alpha-1)(\alpha-2) \cdots\left(\alpha-m_{\beta}\right) \int_{a}^{t}(t-\xi)^{\alpha-m_{\beta}-1} u(\xi) d \xi\right\} \\
= & { }_{a} J_{t}^{m_{\beta}-\beta}\left\{\frac{1}{\Gamma(\alpha)} \frac{(\alpha-1) !}{\left(\alpha-m_{\beta}-1\right) !} \int_{a}^{t}(t-\xi)^{\alpha-m_{\beta}-1} u(\xi) d \xi\right\}
\end{aligned}
$$

Also, using the R-L fractional integral for order $\alpha$ and lemma (1) to obtain:

$$
{ }_{a}^{C} D_{t}^{\beta}\left[{ }_{a} J_{t}^{\alpha} u(t)\right]={ }_{a} J_{t}^{m_{\beta}-\beta}\left[{ }_{a} J_{t}^{\alpha-m_{\beta}} u(t)\right]={ }_{a} J_{t}^{m_{\beta}-\beta+\alpha-m_{\beta}} u(t)={ }_{a} J_{t}^{\alpha-\beta} u(t)
$$

\section{Adomian Decomposition Method (ADM)}

The Adomian Decomposition method, [3,7], consists of decomposing the unknown function $u(t)$ of any equation into a sum of an infinite number of components defined by the decomposition series:

$$
u(t)=u_{0}(t)+u_{1}(t)+\cdots+u_{r}(t)+\cdots=\sum_{r=0}^{\infty} u_{r}(t)
$$

where the components $u_{r}(t), r \geq 0$ are to be determined in a recursive manner. Adomain and Rach [2] and Wazwaz [1] have investigate the phenomena of the self-canceling "noise" terms where the sum of components vanishes in the limit. If the noise terms exist between the components $u_{0}(t)$ and $u_{1}(t)$, it will provide the exact solution by using only the first two iterations. A necessary condition for the appearance of the noise terms is that the zeros component $u_{0}(t)$ must contain the exact solution among the otherterms. first we apply $\alpha_{n}$-order of R-L fractional integral for both sides of our problem and using lemma (1) and lemma (2, part-i) we obtain:

$$
\begin{aligned}
u(t)={ }_{a} J_{t}^{\alpha_{n}} f(t) & +\sum_{k=0}^{m_{\alpha_{n}-1}} \frac{u^{(k)}(a)}{k !}(t-a)^{k}+\sum_{i=1}^{n-1}{ }_{a} J_{t}^{\alpha_{n}}\left[\bar{P}_{i}(t){ }_{a}^{c} D_{t}^{\alpha_{n-i}} u(t)\right]+{ }_{a} J_{t}^{\alpha_{n}}\left[\bar{P}_{n}(t) u(t)\right] \\
& +\lambda \sum_{j=0}^{m} \int_{a}^{b} \overline{\mathcal{K}}_{j}(t, s){ }_{a}^{c} D_{s}^{\beta_{m-j}} u(s) d s
\end{aligned}
$$

where $\bar{P}_{i}(t)=-P_{i}(t)$ for all $i=1,2, \cdots, n$ and $\overline{\mathcal{K}}_{j}(t, s)={ }_{a} J_{t}^{\alpha_{n}} \mathcal{K}_{j}(t, s)$ for all $j=0,1, \cdots, m$.

Second, according to the decomposition method, we assume the series solution for the unknown function $u(t)$ in the form (4) and it is leads to the following recursive relation:

$$
\left.\begin{array}{l}
u_{0}(t)={ }_{a} J_{t}^{\alpha_{n}} f(t)+\sum_{k=0}^{m_{\alpha_{n}-1}} \frac{u^{(k)}(a)}{k !}(t-a)^{k} \\
u_{r+1}(t)=\sum_{i=1}^{n-1} a J_{t}^{\alpha_{n}}\left[\bar{P}_{i}(t){ }_{a}^{c} D_{t}^{\alpha_{n-i}} u_{r}(t)\right]+{ }_{a} J_{t}^{\alpha_{n}}\left[\bar{P}_{n}(t) u_{r}(t)\right] \\
+\lambda \sum_{j=0}^{m} \int_{a}^{b} \overline{\mathcal{K}}_{j}(t, s){ }_{a}^{C} D_{s}^{\beta_{m-j}} u_{r}(s) d s \quad \text {,for all } r \geq 0
\end{array}\right\}
$$

Now, as a special case if $\bar{P}_{i}(t)=-C_{i}=\bar{C}_{i}(i=1,2, \cdots, n-1)$ where $\bar{C}_{i}$ are any real constants then we can write equation (6) as follows: 


$$
\begin{aligned}
& u_{0}(t)={ }_{a} J_{t}^{\alpha_{n}} f(t)+\sum_{k=0}^{m_{\alpha_{n}}-1} \frac{u^{(k)}(a)}{k !}(t-a)^{k} \\
& +\sum_{i=1}^{n-1} C_{i}\left[\sum_{k=0}^{m_{\alpha_{n-i}-1}} \frac{u^{(k)}(a)}{\Gamma\left(k+\alpha_{n}-\alpha_{n-i}+1\right)}(t-a)^{k+\alpha_{n}-\alpha_{n-i}}\right] \\
& u_{r+1}(t)=\sum_{i=1}^{n-1} \bar{C}_{i}\left[a J_{t}^{\alpha_{n}-\alpha_{n-i}} u_{r}(t)\right]+{ }_{a} J_{t}^{\alpha_{n}}\left[\bar{P}_{n}(t) u_{r}(t)\right] \\
& +\lambda \sum_{j=0}^{m}\left[\int_{a}^{b} \overline{\mathcal{K}}_{j}(t, s){ }_{a}^{C} D_{s}^{\beta_{m-j}} u_{r}(s) d s\right], r \geq 0
\end{aligned}
$$

The components $u_{0}, u_{1}, u_{2}, \cdots$ are determined recursively by above formula (6) or (7). It is important to note that the decomposition method suggests that the zeros component $u_{0}(t)$ be defined by the conditions and the function $f(t)$ as described above. The other components namely $u_{1}, u_{2}, \cdots$ are derived recurrently. so we use an approximation of the solution by the following truncated series:

$$
u(t) \cong \hat{u}_{n}(t)=\sum_{r=0}^{n} u_{r}(t), \quad n=0,1,2, \cdots
$$

\section{Modified Adomian Decomposition Method (MADM)}

It is interesting to note that the modified decomposition method depends mainly on splitting the inhomogeneous into finite parts namely $f_{i}(t), i=1,2, \ldots, N \in \mathbb{N}$, therefore it cannot be used if the function consists of only one term. The success of this modification depends only the paper choice of $f_{i}(t)$ and this can be made through trials only.

By the same stages of ADM, we obtain equation (5) as following form:

$$
\begin{gathered}
u(t)=g(t)+\sum_{i=1}^{n-1} a J_{t}^{\alpha_{n}}\left[\bar{P}_{i}(t){ }_{a}^{C} D_{t}^{\alpha_{n-i}} u(t)\right]+{ }_{a} J_{t}^{\alpha_{n}}\left[\bar{P}_{n}(t) u(t)\right] \\
+\lambda \sum_{j=0}^{m} \int_{a}^{b} \overline{\mathcal{K}}_{j}(t, s){ }_{a}^{c} D_{s}^{\beta_{m-j}} u(s) d s
\end{gathered}
$$

where

$$
\begin{gathered}
g(t)={ }_{a} J_{t}^{\alpha_{n}} f(t)+\sum_{k=0}^{m_{\alpha_{n}-1}} \frac{u^{(k)}(a)}{k !}(t-a)^{k}, \quad m_{\alpha_{n}}=\left\lceil\alpha_{n}\right\rceil \\
\overline{\mathcal{K}}_{j}(t, s)={ }_{a} J_{t}^{\alpha_{n}} \mathcal{K}_{j}(t, s) \text { and } \bar{P}_{i}(t)=-P_{i}(t)
\end{gathered}
$$

In this method the function $g(t)$ can be set as the sum of finite partial functions $f_{i}(t), i=0,1, \ldots, N \in \mathbb{N}$. In other words, we can set:

$$
g(t)=f_{0}(t)+f_{1}(t)+\cdots+f_{N}(t)
$$

Here, the zeros component is defined by one part of $g(t)$, namely $f_{0}(t)$ or $f_{1}(t)$ and the other part of $g(t)$ can be added to the component $u_{1}(t)$ among other terms. In other words, the MADM introduces the modified recurrence relation: 


$$
\begin{aligned}
u_{0}(t)= & f_{0}(t) \\
u_{i}(t)= & f_{i}(t)+\sum_{i=1}^{n-1} a J_{t}^{\alpha_{n}}\left[\bar{P}_{i}(t){ }_{a}^{C} D_{t}^{\alpha_{n-i}} u_{0}(t)\right]+{ }_{a} J_{t}^{\alpha_{n}}\left[\bar{P}_{n}(t) u_{0}(t)\right] \\
& +\lambda \sum_{j=0}^{m} \int_{a}^{b} \overline{\mathcal{K}}_{j}(t, s){ }_{a}^{C} D_{s}^{\beta_{m-j}} u_{0}(s) d s, \quad(i \geq 1) \\
u_{r+1}(t)= & \sum_{i=1}^{n-1} a J_{t}^{\alpha_{n}}\left[\bar{P}_{i}(t){ }_{a}^{C} D_{t}^{\alpha_{n-i}} u_{r}(t)\right]+{ }_{a} J_{t}^{\alpha_{n}}\left[\bar{P}_{n}(t) u_{r}(t)\right] \\
& +\lambda \sum_{j=0}^{m} \int_{a}^{b} \overline{\mathcal{K}}_{j}(t, s){ }_{a}^{C} D_{s}^{\beta_{m-j}} u_{r}(s) d s, \quad(r \geq N)
\end{aligned}
$$

Now, as a special case if $\bar{P}_{i}(t)=\bar{C}_{i}$ where $\bar{C}_{i}=-C_{i}=-P_{i}(t)$ for all $i=1,2, \cdots, n-1$ is any constant then $g(t)$ in equation (8) becomes:

$g(t)={ }_{a} J_{t}^{\alpha_{n}} f(t)+\sum_{k=0}^{m_{\alpha_{n}-1}} \frac{u^{(k)}(a)}{k !}(t-a)^{k}+\sum_{i=1}^{n-1} C_{i}\left(\sum_{k=0}^{m_{\alpha_{n-i}-1}} \frac{(t-a)^{k+\alpha_{n}-\alpha_{n-i}}}{\left(k+\alpha_{n}-\alpha_{n-i}+1\right)} u^{(k)}(a)\right)$

by the same way the function $g(t)$ is defined as the sum of finite partial functions $f_{i}(t), i \geq 1$ and the components $u_{0}(t), u_{1}(t), \cdots, u_{r+1}(t)$ is defined as:

$$
\begin{aligned}
& u_{0}(t)=f_{1}(t) \\
& u_{i}(t)=f_{i+1}(t)+\sum_{i=1}^{n-1} \bar{C}_{i a} J_{t}^{\alpha_{n}-\alpha_{n-i}}\left[u_{0}(t)\right]+{ }_{a} J_{t}^{\alpha_{n}}\left[\bar{P}_{n}(t) u_{0}(t)\right] \\
& +\lambda \sum_{j=0}^{m} \int_{a}^{b} \overline{\mathcal{K}}_{j}(t, s){ }_{a}^{c} D_{s}^{\beta_{m-j}} u_{0}(s) d s, \quad(i \geq 1) \\
& u_{r+1}(t)=\sum_{i=1}^{n-1} \bar{C}_{i a} J_{t}^{\alpha_{n}-\alpha_{n-i}}\left[u_{r}(t)\right]+{ }_{a} J_{t}^{\alpha_{n}}\left[\bar{P}_{n}(t) u_{r}(t)\right] \\
& +\lambda \sum_{j=0}^{m} \int_{a}^{b} \overline{\mathcal{K}}_{j}(t, s){ }_{a}^{c} D_{s}^{\beta_{m-j}} u_{r}(s) d s, \quad(r \geq N)
\end{aligned}
$$

so we use an approximation of the solution by the following truncated series:

$$
u(t) \cong \hat{u}_{n}(t)=\sum_{r=0}^{n} u_{r}(t), \quad n=0,1,2, \cdots
$$

5. Illustrate Examples:

Here, two analytical results were presented for linear IFDE of Fredholm type with variable coefficients using Adomian Decomposition and Modified Adomian Decomposition method.

\section{Example (1)}

Consider the linear Fredholm of IFDE with variable coefficients for multi-higher fractional orders:

${ }_{0}^{C} D_{t}^{0.8} u(t)+t^{2}{ }_{0}^{C} D_{t}^{0.6} u(t)-3 u(t)=f(t)$

where

$$
+\int_{0}^{1}\left[{ }_{0}^{C} D_{s}^{0.5} u(s)+\left(t+s^{2}\right){ }_{0}^{C} D_{s}^{0.2} u(s)\right] d s
$$

$$
f(t)=\frac{-6}{\Gamma(2.2)} t^{1.2}-\frac{6}{\Gamma(2.4)} t^{3.4}+9 t^{2}+\frac{6}{\Gamma(3.8)} t+\frac{6}{\Gamma(3.5)}+\frac{6}{4.8 \Gamma(2.8)}
$$


subjected to the condition $u(0)=0$. Applying the ADM for solving our problem, from first part of equation (6) we obtain:

$$
u_{0}(t)=-3 t^{2}-\frac{6 \Gamma(4.4)}{\Gamma(2.4) \Gamma(5.2)} t^{4.2}+\frac{18}{\Gamma(3.8)} t^{2.8}+\frac{6}{\Gamma(3.8) \Gamma(2.8)} t^{1.8}+\frac{6}{\Gamma(1.8)}\left[\frac{1}{\Gamma(3.5)}+\frac{1}{4.8 \Gamma(2.8)}\right] t^{0.8}
$$

For the second part of equation (6) putting $r=0$ in it and finding each parts we get $u_{1}(t)$ as follows:

$$
\begin{aligned}
& u_{1}(t)=\frac{6 \Gamma(4.4)}{\Gamma(2.4) \Gamma(5.2)} t^{4.2}+\frac{6 \Gamma(4.4) \Gamma(6.6)}{\Gamma(2.6) \Gamma(4.6) \Gamma(7.4)} t^{6.4}-\left[\frac{3 \Gamma(5.2)}{20 \Gamma(3.2)}+\frac{3 \Gamma(4.4)}{20 \Gamma(2.4)}\right] t^{5}+\frac{\Gamma(4.2)}{4 \Gamma(3.8) \Gamma(2.2)} t^{4} \\
&+\frac{\Gamma(3.2)}{\Gamma(1.2)} t^{3}-\frac{18}{\Gamma(3.8)} t^{2.8}+\frac{54}{\Gamma(4.6)} t^{3.6}+\frac{18}{\Gamma(3.6) \Gamma(3.8)} t^{2.6} \\
&+\frac{18}{\Gamma(2.6)} t^{1.6}\left[\frac{1}{\Gamma(3.5)}+\frac{1}{4.8 \Gamma(2.8)}\right] \\
&+\frac{1}{\Gamma(2.8)} t^{1.8}\left[\frac{-6}{\Gamma(3.8)}-\frac{\Gamma(4.4)}{5 \Gamma(2.4)}+\frac{6}{\Gamma(3.8) \Gamma(3.6)}+\frac{18}{\Gamma(4.6)}\right. \\
&\left.+\frac{6}{\Gamma(2.6)}\left(\frac{1}{\Gamma(3.5)}+\frac{1}{4.8 \Gamma(2.8)}\right)\right] \\
&+\frac{1}{\Gamma(1.8)} t^{0.8}\left[\frac{-6}{\Gamma(3.5)}-\frac{6 \Gamma(4.4)}{\Gamma(2.4) \Gamma(5.7)}+\frac{18}{\Gamma(4.3)}+\frac{6}{3.8 \Gamma(3.3)}\right. \\
&+\frac{6}{\Gamma(2.3)}\left(\frac{1}{\Gamma(3.5)}+\frac{1}{4.8 \Gamma(2.8)}\right)-\frac{6}{4.8 \Gamma(2.8)}-\frac{\Gamma(4.4)}{7 \Gamma(2.4)}+\frac{6}{4.6 \Gamma(2.6) \Gamma(3.8)}+\frac{18}{5.6 \Gamma(3.6)} \\
&\left.+\frac{6}{3.6 \Gamma(1.6)}\left(\frac{1}{\Gamma(3.5)}+\frac{1}{4.8 \Gamma(2.8)}\right)\right]
\end{aligned}
$$

The noise terms:

$$
\pm \frac{6 \Gamma(4.4)}{\Gamma(2.4) \Gamma(5.2)} t^{4.2} \pm \frac{18}{\Gamma(3.8)} t^{2.8} \pm \frac{6}{\Gamma(3.8) \Gamma(2.8)} t^{1.8} \pm \frac{6}{\Gamma(1.8)} t^{0.8}\left[\frac{1}{\Gamma(3.5)}+\frac{1}{4.8 \Gamma(2.8)}\right]
$$

appear in $u_{0}(t)$ and $u_{1}(t)$. Cancelling this terms from the zeros component $u_{0}(t)$ gives the solution $u(t) \cong \hat{u}_{1}(t)=-3 t^{2}$ which is the exact solution that satis fies our linear FIFDE's.

\section{Example (2)}

Consider we have the following linear IFDE of Fredholm type:

${ }_{0}^{C} D_{t}^{1.6} u(t)-5{ }_{0}^{C} D_{t}^{1.3} u(t)+t u(t)=f(t)+\int_{0}^{1}\left[(t-s){ }_{0}^{C} D_{s}^{0.8} u(s)+2 t s{ }_{0}^{C} D_{s}^{0.2} u(s)\right] d s$

where

$$
f(t)=t^{3}-\frac{10}{\Gamma(1.7)} t^{0.7}+\frac{2}{\Gamma(1.4)} t^{0.4}-\left[\frac{2}{\Gamma(3.2)}+\frac{4}{3.8 \Gamma(2.8)}-1\right] t+\frac{2}{3.2 \Gamma(2.2)}
$$

subject to the conditions: $u(0)=1$ and $u^{\prime}(0)=0$,

Using equation (11) we get:

$$
\begin{gathered}
g(t)=t^{2}-\frac{10}{\Gamma(3.3)} t^{2.3}+\frac{6}{\Gamma(5.6)} t^{4.6}-\frac{t^{2.6}}{\Gamma(3.6)}\left[\frac{2}{\Gamma(3.2)}+\frac{4}{3.8 \Gamma(2.8)}-1\right]+\frac{2}{3.2 \Gamma(2.2) \Gamma(2.6)} t^{1.6}+1 \\
-\frac{5}{\Gamma(1.3)} t^{0.3}
\end{gathered}
$$

From $g(t)$ we assume that:

$$
\begin{gathered}
f_{1}(t)=t^{2}+1 \\
f_{2}(t)=-\frac{10}{\Gamma(3.3)} t^{2.3}+\frac{6}{\Gamma(5.6)} t^{4.6}-\frac{t^{2.6}}{\Gamma(3.6)}\left[\frac{2}{\Gamma(3.2)}+\frac{4}{3.8 \Gamma(2.8)}-1\right]+\frac{2}{3.2 \Gamma(2.2) \Gamma(2.6)} t^{1.6} \\
-\frac{5}{\Gamma(1.3)} t^{0.3}
\end{gathered}
$$

We next use the MADM recurrence formula (12) to obtain:

$u_{0}(t)=f_{1}(t)=t^{2}+1$ 


$$
\begin{aligned}
u_{1}(t)=f_{2}(t)+ & 5{ }_{0} J_{t}^{0.3}\left[u_{0}(t)\right]-{ }_{0} J_{t}^{1.6}\left[t u_{0}(t)\right]+\int_{0}^{1}\left[{ }_{0} J_{t}^{1.6}[t-s]{ }_{0}^{c} D_{s}^{0.8} u_{0}(s)+{ }_{0} J_{t}^{1.6}[2 t s]{ }_{0}^{c} D_{s}^{0.2} u_{0}(s)\right] d s \\
= & 0
\end{aligned}
$$

It follows immediately that

$$
u_{r+1}(t)=0, \forall r \geq 1
$$

So $u(t)=t^{2}+1$ is the solution which is the exact expression for our linear FIFDE.

Example (3)

Consider the higher order linear FIFDE with variable coefficients:

$$
{ }_{0}^{C} D_{t}^{1.9} u(t)+t u(t)=f(t)+\int_{0}^{1} 2 t s\left[{ }_{0}^{C} D_{s}^{0.7} u(s)\right] d s
$$

where

$$
f(t)=\frac{4}{3.3 \Gamma(2.3)} t-\frac{2}{\Gamma(1.1)} t^{0.1}-t^{3}
$$

subjected to the conditions: $u(0)=0, u^{\prime}(0)=0$, while the exact solution is: $u(t)=-t^{2}$.

First apply standard ADM for solving our problem. Here, $n=1, m=0, \alpha_{1}=1.9, \beta_{0}=0.7, P_{1}(t)=t$ and $\mathcal{K}_{0}(t, s)=2 t s$. From first part of equation (6) we obtain:

$$
u_{0}(t)=-t^{2}+\frac{4}{3.3 \Gamma(2.3) \Gamma(3.9)} t^{2.9}-\frac{6}{\Gamma(5.9)} t^{4.9}
$$

For the second part of equation (6) putting $r=0$ in it and finding each parts we get $u_{1}(t)$ as follows:

$$
\begin{aligned}
u_{1}(t)={ }_{a} J_{t}^{\alpha_{1}}\left[\bar{P}_{1}(t)\right. & \left.u_{0}(t)\right]+\lambda \sum_{j=0}^{m} \int_{0}^{1} \overline{\mathcal{K}}_{0}(t, s){ }^{c} D_{s}^{\beta_{0}} u_{0}(s) d s \\
& =\frac{6 \Gamma(6.9)}{\Gamma(5.9) \Gamma(8.8)} t^{7.8}-\frac{4 \Gamma(4.9)}{3.3 \Gamma(2.3) \Gamma(3.9) \Gamma(6.8)} t^{5.8}+\frac{6}{\Gamma(5.9)} t^{4.9}-\frac{4}{3.3 \Gamma(2.3) \Gamma(3.9)} t^{2.9}
\end{aligned}
$$

so the first approximation of the solution is

$$
u(t) \cong \hat{u}_{1}(t)=u_{0}(t)+u_{1}(t)=-t^{2}+\frac{6 \Gamma(6.9)}{\Gamma(5.9) \Gamma(8.8)} t^{7.8}-\frac{4 \Gamma(4.9)}{3.3 \Gamma(2.3) \Gamma(3.9) \Gamma(6.8)} t^{5.8}
$$

Putting $r=1$, into equation (6) for obtaining $u_{2}(t)$. Thus:

$$
\begin{gathered}
u_{2}(t)={ }_{a} J_{t}^{\alpha_{1}}\left[\bar{P}_{1}(t) u_{1}(t)\right]+\lambda \sum_{j=0}^{m} \int_{0}^{1} \overline{\mathcal{K}}_{0}(t, s) \quad{ }^{c} D_{s}^{\beta_{0}} u_{1}(s) d s \\
=\frac{27.2 \Gamma(4.9)}{3.3 \Gamma(2.3) \Gamma(3.9) \Gamma(9.7)} t^{8.7}-\frac{35.4}{\Gamma(8.8)} t^{7.8}-\frac{52.8 \Gamma(6.9)}{\Gamma(5.9) \Gamma(11.7)} t^{10.7}+\frac{15.6}{3.3 \Gamma(2.3) \Gamma(6.8)} t^{5.8} \\
+\frac{2 t^{2.9}}{\Gamma(3.9)}\left[\frac{-4 \Gamma(4.9)}{23.43 \Gamma(2.3) \Gamma(3.9) \Gamma(6.1)}+\frac{6}{6.2 \Gamma(5.2)}+\frac{6 \Gamma(6.9)}{9.1 \Gamma(5.9) \Gamma(8.1)}\right. \\
\left.-\frac{4}{13.86 \Gamma(2.3) \Gamma(3.2)}\right]
\end{gathered}
$$

so the second approximation of the solution is

$$
\begin{aligned}
& =-t^{2}+\frac{27.2 \Gamma(4.9)}{3.3 \Gamma(2.3) \Gamma(3.9) \Gamma(9.7)} t^{8.7}-\frac{52.8 \Gamma(6.9)}{\Gamma(5.9) \Gamma(11.7)} t^{10.7} \\
& \quad+\frac{2 t^{2.9}}{\Gamma(3.9)}\left[\frac{-4 \Gamma(4.9)}{23.43 \Gamma(2.3) \Gamma(3.9) \Gamma(6.1)}+\frac{6}{6.2 \Gamma(5.2)}+\frac{6 \Gamma(6.9)}{9.1 \Gamma(5.9) \Gamma(8.1)}\right. \\
& \left.\quad-\frac{4}{13.86 \Gamma(2.3) \Gamma(3.2)}\right]
\end{aligned}
$$

Putting $r=2$, into equation (6) for obtaining $u_{3}(t)$. Thus:

$$
u_{3}(t)={ }_{a} J_{t}^{\alpha_{1}}\left[\bar{P}_{1}(t) u_{2}(t)\right]+\sum_{j=0}^{m} \int_{0}^{1} \overline{\mathcal{K}}_{0}(t, s){ }^{c} D_{s}^{\beta_{0}} u_{2}(s) d s
$$




$$
\begin{aligned}
=\frac{6}{\Gamma(5.9)} t^{4.9}- & \frac{27.2 \Gamma(4.9) \Gamma(10.7)}{3.3 \Gamma(2.3) \Gamma(3.9) \Gamma(9.7) \Gamma(12.6)} t^{11.6}+\frac{52.8 \Gamma(6.9) \Gamma(12.7)}{\Gamma(5.9) \Gamma(11.7) \Gamma(14.6)} t^{13.6} \\
& -\frac{2 \Gamma(4.9)}{\Gamma(3.9) \Gamma(6.8)} t^{5.8}\left[\frac{6}{23.43 \Gamma(2.3) \Gamma(3.9) \Gamma(6.1)}+\frac{6}{6.2 \Gamma(5.2)}+\frac{6 \Gamma(6.9)}{9.1 \Gamma(5.9) \Gamma(8.1)}\right. \\
& \left.-\frac{4}{13.86 \Gamma(2.3) \Gamma(3.2)}\right] \\
& +\frac{2 t^{2.9}}{\Gamma(3.9)}\left[\frac{-2}{3.2 \Gamma(2.3)}+\frac{27.2 \Gamma(4.9) \Gamma(9.7)}{33 \Gamma(2.3) \Gamma(3.9) \Gamma(9.7) \Gamma(9)}-\frac{52.8 \Gamma(6.9) \Gamma(11.7)}{12 \Gamma(5.9) \Gamma(11.7) \Gamma(11)}\right. \\
& +\frac{2}{4.2 \Gamma(3.2)}\left(\frac{6 \Gamma(6.9)}{23.43 \Gamma(2.3) \Gamma(3.9) \Gamma(6.1)}+\frac{6}{6.2 \Gamma(5.2)}+\frac{6 \Gamma(5.9)}{9.1 \Gamma(5.9) \Gamma(8.1)}\right. \\
& \left.\left.-\frac{4}{13.86 \Gamma(2.3) \Gamma(3.2)}\right)\right]
\end{aligned}
$$

so the second approximation of the solution is

$$
\begin{aligned}
& u(t) \cong \hat{u}_{3}(t)=u_{0}(t)+u_{1}(t)+u_{2}(t)+u_{3}(t) \\
& =-t^{2}+\frac{27.2 \Gamma(4.9)}{3.3 \Gamma(2.3) \Gamma(3.9) \Gamma(9.7)} t^{8.7}-\frac{52.8 \Gamma(6.9)}{\Gamma(5.9) \Gamma(11.7)} t^{10.7}+\frac{6}{\Gamma(5.9)} t^{4.9} \\
& -\frac{27.2 \Gamma(4.9) \Gamma(10.7)}{3.3 \Gamma(2.3) \Gamma(3.9) \Gamma(9.7) \Gamma(12.6)} t^{11.6}+\frac{52.8 \Gamma(6.9) \Gamma(12.7)}{\Gamma(5.9) \Gamma(11.7) \Gamma(14.6)} t^{13.6} \\
& -\frac{2 \Gamma(4.9)}{\Gamma(3.9) \Gamma(6.8)} t^{5.8}\left[\frac{-4 \Gamma(4.9)}{23.43 \Gamma(2.3) \Gamma(3.9) \Gamma(6.1)}+\frac{6}{6.2 \Gamma(5.2)}+\frac{6 \Gamma(6.9)}{9.1 \Gamma(5.9) \Gamma(8.1)}\right. \\
& \left.-\frac{4}{13.86 \Gamma(2.3) \Gamma(3.2)}\right] \\
& +\frac{2 t^{2.9}}{\Gamma(3.9)}\left[\frac{-4 \Gamma(4.9)}{23.43 \Gamma(2.3) \Gamma(3.9) \Gamma(6.1)}+\frac{6}{6.2 \Gamma(5.2)}+\frac{6 \Gamma(6.9)}{9.1 \Gamma(5.9) \Gamma(8.1)}\right. \\
& -\frac{4}{13.86 \Gamma(2.3) \Gamma(3.2)}+\frac{-2}{3.2 \Gamma(2.3)}+\frac{27.2 \Gamma(4.9) \Gamma(9.7)}{33 \Gamma(2.3) \Gamma(3.9) \Gamma(9.7) \Gamma(9)} \\
& -\frac{52.8 \Gamma(6.9) \Gamma(11.7)}{12 \Gamma(5.9) \Gamma(11.7) \Gamma(11)} \\
& +\frac{2}{4.2 \Gamma(3.2)}\left(\frac{-4 \Gamma(4.9)}{23.43 \Gamma(2.3) \Gamma(3.9) \Gamma(6.1)}+\frac{6}{6.2 \Gamma(5.2)}+\frac{6 \Gamma(6.9)}{9.1 \Gamma(5.9) \Gamma(8.1)}\right. \\
& \left.\left.-\frac{4}{13.86 \Gamma(2.3) \Gamma(3.2)}\right)\right]
\end{aligned}
$$

gives the solution $u(t)=-t^{2}$ which is the exact solution that satis fies our linear FIFDE's.

\section{Second apply MADM,}

Using equation (11) we get:

From $g(t)$ we assume that:

$$
g(t)=-t^{2}+\frac{4}{3.3 \Gamma(2.3) \Gamma(3.9)} t^{2.9}-\frac{6}{\Gamma(5.9)} t^{4.9}
$$

$$
f_{1}(t)=-t^{2}, f_{2}(t)=\frac{4}{3.3 \Gamma(2.3) \Gamma(3.9)} t^{2.9}-\frac{6}{\Gamma(5.9)} t^{4.9}
$$

We next use the MADM recurrence formula (12) to obtain:

$u_{0}(t)=f_{1}(t)=-t^{2}$

$u_{1}(t)=f_{2}(t)+{ }_{0} J_{t}^{1.9}\left[(-t) u_{0}(t)\right]+\int_{0}^{1}\left[{ }_{0} J_{t}^{1.9}[2 t s]{ }_{0}^{c} D_{s}^{0.7} u_{0}(s)\right] d s=0$

It follows immediately that

$$
u_{r+1}(t)=0, \forall r \geq 1
$$

So $u(t)=-t^{2}$ is the solution which is the exact expression for our linear FIFDE. 
6. Conclusion

In this paper, two analytic and approximate methods for treating "linear integro-fractional differential equation of Fredholm type with variable coefficients" were introduced with some illustrating examples for each method and the following points have been noticed:

1. In general, these analytical and approximate methods proposed here provided good results and effectiveness in solving our problem.

2. Sometimes the Noise terms in Adomian method will not appear, so we use modified Adomian Decomposition method.

\section{References}

1. Abdul-Majid Wazwaz (2011) Linear and Nonlinear Integral Equations, Methods and Application, Springer Heidelberg Dordrecht London, New York.

2. Adomian, G. and Rach, R. (1992), "Noise Terms in Decomposition Solution Series" Computers Math. Applic.,24(11): 61-64.

3. Adomian, G. and Rach, R. (1993) Analytic Solution of Nonlinear Boundary Value Problems in Several Dimensions by Decomposition. Journal of Mathematical Analysis and Applications, 174,118-137.

4. Anatoly, A. Kilbas, Hari M. Srivastava and Juan J. Trujillo, (2006), Theory and Applications of Fractional Differential Equations, Elsevier B.V. Netherlands.

5. Dogan Kaya (2002), the use of Adomian decomposition method for solving a specific nonlinear partial differential equation, Bull. Belg. Math Soc. 9, pp 343-349.

6. Ercan Celik, Mustafa Bayram and Turgut Yeloglu (2006) Solution of Differential-Algebraic Equations(DAES) by Adomian Decomposition Method, International Journal Pure \& Applied Mathematical Sciences, Vol.3 No.1, pp93-100.

7. George Adomian, (1994) "Solv ing Frontier Problems of Physics: The Decomposition Method" Kluwer Academic Publishers, Boston.

8. Hossein Jafari and Varsha Daftardar-Gejji (2006), Solving a system of nonlinear fractional differential equations using Adomian decomposition, Journal of Computational and applied Mathematics, 196, pp 644-651.

9. I. podldubny, (1999) “Fractional Differential Equation, Academic press" San Diego.

10. Kai Diethelm, (2010), The Analysis of Fractional Differential Equations Springer-Verlag Berlin Heidelberg.

11. Kelth, B. Oldham and Jerome Spanier, (1974), The Fractional Calculus: Theory and applications of differentiation and integration to arbitrary order, Academic press, Inc.

12. Kenneth S. Miller and Bertram Ross, (1993) "An Introduction to the Fractional Calculus and Fractional Differential Equations" John Wiley \& Sons (New York).

13. Marc Weilbeer, (2005) "Efficient Numerical Methods for Fractional Differential Equations and their Analytical Background", US Army Medical Research and Material Command.

14. M. Javidi and A. Golbabai (2007) Adomian Decomposition Method for Approximating the Solution of the Parabolic Equations, Applied Mathematical Sciences, Vol. 1, No. 5, pp 219-225.

15. Shazad Shawki Ahmed, (2009), "On System of Linear Volterra Integro- Fractional Differential Equations" Ph.D. Thesis, Sulaimani University. 